\title{
The Convergences Comparison between the Halley's Method and Its Extended One Based on Formulas Derivation and Numerical Calculations
}

\author{
Shunji Horiguchi \\ Department of Economics, Niigata Sangyo University, Niigata, Japan \\ Email: shori@econ.nsu.ac.jp
}

How to cite this paper: Horiguchi, S. (2016) The Convergences Comparison between the Halley's Method and Its Extended One Based on Formulas Derivation and Numerical Calculations. Applied Mathematics, 7, 23942410.

http://dx.doi.org/10.4236/am.2016.718188

Received: October 22, 2016

Accepted: December 24, 2016

Published: December 27, 2016

Copyright $\odot 2016$ by author and Scientific Research Publishing Inc. This work is licensed under the Creative Commons Attribution International License (CC BY 4.0).

http://creativecommons.org/licenses/by/4.0/

\begin{abstract}
The purpose of this paper is that we give an extension of Halley's method (Section 2), and the formulas to compare the convergences of the Halley's method and extended one (Section 3). For extension of Halley's method we give definition of function by variable transformation in Section 1. In Section 4 we do the numerical calculations of Halley's method and extended one for elementary functions, compare these convergences, and confirm the theory. Under certain conditions we can confirm that the extended Halley's method has better convergence or better approximation than Halley's method.
\end{abstract}

\section{Keywords}

Recurrence Formula, Newton's Method, Halley's Method, Extension of Halley's Method, Third-Order Convergence

\section{Introduction}

In 1673, Yoshimasu Murase [1] made a cubic equation to obtain the thickness of a hearth. He introduced two kinds of recurrence formulas of square $x_{k}^{2}$ and the deformation. We find that the three formulas lead to a Horner's method (Horiguchi, [2]) and an extension of Newton's method (Horiguchi, [3]). This shows originality of Wasan (mathematics developed in Japan) in the Edo era (1603-1868). We do research similar to Horiguchi, [3] against the Halley's method. We give function $y=g(t)$ defined from $f(x)$ for extension of Halley's method.

From now on, let $x$ be a real number, and a function $f(x) i(\geq 1)$ times differentiable if necessary, and $f^{(i)}(x)$ continuous.

Definition 1.1. Let $x^{q}=t$ where $q$ is a real number that is not 0 . We define the 
function $g(t)$ such as

$$
g(t):=f\left(t^{1 / q}\right)=f(x) .
$$

Because $g\left(x^{q}\right)=f(x)$, the graph of $g(x)$ extends or contracts by $x^{q}=t$ in the $x$-axis, without changing the height of $y=f(x)$. Expansion and contraction come to object in $|x|<1$ and $|x|>1$.

Theorem 1.2. The formulas

$$
g^{\prime \prime}(t)=g^{\prime \prime}\left(x^{q}\right)=\left\{\begin{array}{l}
\frac{x f^{\prime \prime}(x)+(1-q) f^{\prime}(x)}{q^{2} x^{2 q-1}}>0(<0 \text { resp. }), x \neq 0 \\
\frac{f^{\prime \prime}(x)\left(1+\frac{1-q}{x} \frac{f^{\prime}(x)}{f^{\prime \prime}(x)}\right)}{\left(q x^{q-1}\right)^{2}}>0(<0 \text { resp. }), x \neq 0, f^{\prime \prime}(x) \neq 0
\end{array}\right.
$$

give the convex upward (the convex downward resp.) at the point $x^{q}$ of graph of $g(x)$.

Proof. It is proved by the next calculations.

$$
\begin{gathered}
g^{\prime}(t)=\frac{d g(t)}{d t}=\frac{d f(x)}{d t}=\frac{d f(x)}{d x} \frac{d x}{d t}=f^{\prime}(x) \cdot \frac{1}{q x^{q-1}} \\
g^{\prime \prime}(t)=\frac{d g^{\prime}(t)}{d t}=\frac{d}{d t} \frac{f(x)}{q x^{q-1}}=\frac{d}{d x} \frac{f^{\prime}(x)}{q x^{q-1}} \frac{d x}{d t}=\frac{f^{\prime \prime}(x) q x^{q-1}-f^{\prime}(x)\left(q x^{q-1}\right)^{\prime}}{\left(q x^{q-1}\right)^{2}} \frac{1}{q x^{q-1}}
\end{gathered}
$$

From the formulas (4), (5), we obtain the next theorem.

Theorem 1.3. The curvature of the cure $y=g(x)$ at the point $x^{q}$ is formulas (6) and (7).

$$
\mu_{q}(t)=\frac{g^{\prime \prime}(t)}{\left(1+g^{\prime}(t)^{2}\right)^{3 / 2}}=\mu_{q}\left(x^{q}\right)=\left\{\begin{array}{l}
\frac{x f^{\prime \prime}(x)+(1-q) f^{\prime}(x)}{q^{2} x^{2 q-1}\left(1+\left(\frac{f^{\prime}(x)}{q x^{q-1}}\right)^{2}\right)^{3 / 2}}, x \neq 0 \\
\frac{f^{\prime \prime}(x)\left(1+\frac{1-q}{x} \frac{f^{\prime}(x)}{f^{\prime \prime}(x)}\right)}{\left(q x^{q-1}\right)^{2}\left(1+\left(\frac{f^{\prime}(x)}{q x^{q-1}}\right)^{2}\right)^{3 / 2}}, x \neq 0, f^{\prime \prime}(x) \neq 0
\end{array}\right.
$$

These become the curvature $\mu(x)=\frac{f^{\prime \prime}(x)}{\left(1+f^{\prime}(x)^{2}\right)^{3 / 2}}$ of $f(x)$ if $q=1$ in particular.

Proof. Formula (6) is obtained by substituting the formulas (4) and (5) for $g^{\prime}(t), g^{\prime \prime}(t)$ in the curvature $\mu_{q}(t)$.

Theorem 1.4. A necessary and sufficient condition for 


$$
\left|\mu_{q}\left(x^{q}\right)\right|=\frac{\left|f^{\prime \prime}(x)+\frac{(1-q) f^{\prime}(x)}{x}\right|}{\left(q x^{q-1}\right)^{2}\left(1+\left(\frac{f^{\prime}(x)}{q x^{q-1}}\right)^{2}\right)^{3 / 2}} \leq \frac{\left|f^{\prime \prime}(x)\right|}{\left(1+f^{\prime}(x)^{2}\right)^{3 / 2}}=|\mu(x)|(x \neq 0)
$$

is that formula (9) holds.

$$
\left|1+\frac{f^{\prime}(x)}{f^{\prime \prime}(x)} \frac{1-q}{x}\right| \leq \frac{\left(q x^{q-1}\right)^{2}\left(1+\left(\frac{f^{\prime}(x)}{q x^{q-1}}\right)^{2}\right)^{3 / 2}}{\left(1+f^{\prime}(x)^{2}\right)^{3 / 2}}
$$

Proof. Formula (9) is obtained from (8).

Proposition 1.5. If $\alpha$ is a simple root $(m(>1)$ multiple root resp.) of $f(x)=0$, then $\alpha^{q}$ becomes the simple root ( $m$ multiple root resp.) of $g(x)$.

\section{Halley's Method and Extension of Halley's Method}

Definition 2.1. The recurrence formula to approximate a root of the equation $f(x)=0$

$$
x_{k+1}=x_{k}-\frac{f\left(x_{k}\right)}{f^{\prime}\left(x_{k}\right)-\frac{1}{2} \frac{f\left(x_{k}\right) f^{\prime \prime}\left(x_{k}\right)}{f^{\prime}\left(x_{k}\right)}}, \quad(k=0,1,2, \cdots)
$$

is called Halley's method ${ }^{1}$.

Halley's method is obtained by improving the Newton's method (11) (Ref. [5]).

$$
x_{k+1}=x_{k}-\frac{f\left(x_{k}\right)}{f^{\prime}\left(x_{k}\right)}, \quad(k=0,1,2, \cdots)
$$

They are methods of giving the initial value $x_{0}$, calculating $x_{1}, x_{2}, \cdots$ one after another, and to determine for a root.

From now on we omit the notation $(k=0,1,2, \cdots)$ in recurrence formulas. Applying the Halley's method to $g(t)$, we get

$$
t_{k+1}=t_{k}-\frac{g\left(t_{k}\right)}{g^{\prime}\left(t_{k}\right)-\frac{1}{2} \frac{g\left(t_{k}\right) g^{\prime \prime}\left(t_{k}\right)}{g^{\prime}\left(t_{k}\right)}} .
$$

If we express this by formula (1) in $f\left(x_{k}\right)$, then we get the next definition.

Definition 2.2. Let $\alpha$ be a root of the equation $f(x)=0$. (13) is the recurrence formula to approximate $\alpha^{q}$. We call this the $q$-th power of the extension of Halley's method ( $E H$-method).

$$
x_{k+1}^{q}=x_{k}^{q}-q x_{k}^{q-1} \frac{f\left(x_{k}\right)}{f^{\prime}\left(x_{k}\right)-\frac{1}{2} \frac{f\left(x_{k}\right)\left(\frac{1}{q x_{k}^{q-1}} f^{\prime \prime}\left(x_{k}\right)+(1-q) \frac{1}{x_{k}} f^{\prime}\left(x_{k}\right)\right)}{f^{\prime}\left(x_{k}\right)}}
$$

Here, if $q=1$ then the formula (13) becomes Halley's method.

${ }^{1}$ Edmond Halley (1656-1742) is the British astronomer, and famous for Halley's comet of research. 
Calculation formula of $q$-th power of $E H$-method is this.

$$
x_{k+1}=\left[x_{k}^{q}-q x_{k}^{q-1} \frac{f\left(x_{k}\right)}{f^{\prime}\left(x_{k}\right)-\frac{1}{2} \frac{f\left(x_{k}\right)\left(\frac{1}{q x_{k}^{q-1}} f^{\prime \prime}\left(x_{k}\right)+(1-q) \frac{1}{x_{k}} f^{\prime}\left(x_{k}\right)\right)}{f^{\prime}\left(x_{k}\right)}}\right]^{\frac{1}{q}}
$$

\section{Formulas to Compare the Convergences for Extensions of Halley's Method}

Theorem 3.1. Let $\alpha$ be a simple root for $f(x)=0$, i.e., $f^{\prime}(\alpha) \neq 0$. Then Halley's method becomes the following third-order convergence.

$$
x_{k+1}-\alpha \fallingdotseq \frac{\frac{1}{2} f^{\prime \prime}(\alpha)^{2}-\frac{1}{3} f^{\prime}(\alpha) f^{\prime \prime \prime}(\alpha)}{2 f^{\prime}(\alpha)^{2}}\left(x_{k}-\alpha\right)^{3}
$$

If $\alpha$ is $m(m(\geq 2))$ multiple root, then it becomes the following linearly convergence.

$$
x_{k+1}-\alpha \fallingdotseq\left(1-\frac{2}{m+1}\right)\left(x_{k}-\alpha\right)
$$

Proof. There is a brief proof of (15) in wikipedia [4]. Therefore we go to the proof of (16).

We merely sketch $f(x), f^{\prime}(x), f^{\prime \prime}(x)$ with $f, f^{\prime}, f^{\prime \prime}$. Since $f(x)$ is represented as

$$
f(x)=(x-\alpha)^{m} h(x), \quad h(\alpha) \neq 0,
$$

$f^{\prime}, f^{\prime \prime}, f^{\prime 2}, f f^{\prime \prime}$ is as follows, respectively.

$$
\left\{\begin{array}{l}
f^{\prime}=m(x-\alpha)^{m-1} h+(x-\alpha)^{m} h^{\prime} \\
f^{\prime \prime}=(x-\alpha)^{m-2}\left(m(m-1) h+2 m h^{\prime}(x-\alpha)+(x-\alpha)^{2} h^{\prime \prime}\right) \\
f^{\prime 2}=m^{2}(x-\alpha)^{2 m-2} h^{2}+2 m(x-\alpha)^{2 m-1} h h^{\prime}+(x-\alpha)^{2 m} h^{\prime 2} \\
f^{\prime \prime}=h(x-\alpha)^{2 m-2}\left(m(m-1) h+2 m h^{\prime}(x-\alpha)+h^{\prime \prime}(x-\alpha)^{2}\right)
\end{array}\right.
$$

From these formulas, we obtain the following linearly convergence.

$$
\begin{aligned}
x_{k+1}-\alpha= & x_{k}-\alpha-\frac{2 f f^{\prime}}{2 f^{\prime 2}-f f^{\prime \prime}}=x_{k}-\alpha \\
& -\frac{h\left(x_{k}-\alpha\right)^{m}\left(2 m h+2 h^{\prime}\left(x_{k}-\alpha\right)\right)}{\left(m^{2} h^{2}+m h^{2}\right)\left(x_{k}-\alpha\right)^{m-1}+2 m h h^{\prime}\left(x_{k}-\alpha\right)^{m}+\left(2 h^{\prime 2}-h h^{\prime \prime}\right)\left(x_{k}-\alpha\right)^{m+1}} \\
= & x_{k}-\alpha-\frac{2 m h^{2}}{m^{2} h^{2}+m h^{2}}\left(x_{k}-\alpha\right) \\
= & \left(1-\frac{2}{m+1}\right)\left(x_{k}-\alpha\right)
\end{aligned}
$$

Lemma 3.2. In the sequence $\left\{x_{n}\right\}$, let $\lim _{n \rightarrow \infty} x_{n}=\alpha$, and $q, r$ an arbitrary real constant number that is not 0 , respectively. In this case following formula holds for 
large enough integer $n$.

$$
x_{n}^{q}-\alpha^{q} \fallingdotseq \frac{q}{r} \alpha^{q-r}\left(x_{n}^{r}-\alpha^{r}\right)
$$

Proof. Applying L'Hospital's rule to $\left(x^{q}-\alpha^{q}\right) /\left(x^{r}-\alpha^{r}\right)$, formula (20) is obtained.

Theorem 3.3. Let the condition be the same as Theorem 3.1. If $X_{k}$ sufficiently close to $\alpha(\neq 0)$, then $q$-th power of $E H$-method (Extended Halley's method) becomes the third-order convergence of the following formula.

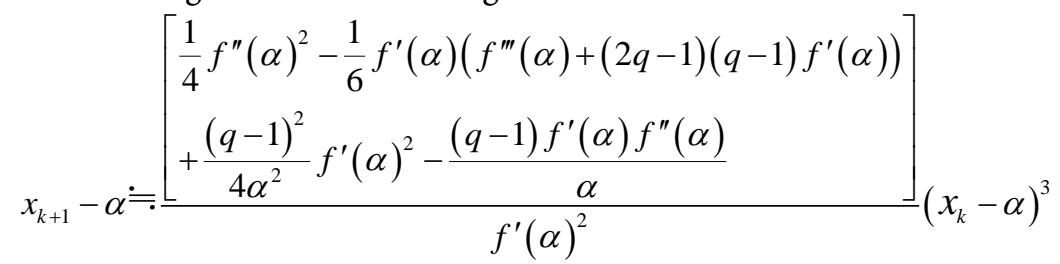

If $\alpha$ is $m(m(\geq 2))$ multiple root, then it will be linearly convergence of the following formula.

$$
x_{k+1}-\alpha \fallingdotseq\left(1-\frac{2}{m+1}\right)\left(x_{k}-\alpha\right)
$$

Proof. If $\alpha$ is a simple root for $f(x)=0$, then $t=\alpha^{q}$ also becomes a simple root for $g(t)=0$. In this case Halley's method for $g(t)$ becomes the third-order convergence of the following formula.

$$
t_{k+1}-\alpha^{q} \fallingdotseq \frac{\frac{1}{4} g^{\prime \prime}\left(\alpha^{q}\right)^{2}-\frac{1}{6} g^{\prime}\left(\alpha^{q}\right) g^{\prime \prime \prime}\left(\alpha^{q}\right)}{g^{\prime}\left(\alpha^{q}\right)^{2}}\left(t_{k}-\alpha^{q}\right)^{3}
$$

Here $g^{\prime}, g^{\prime 2}, g^{\prime \prime}, g^{\prime \prime 2}, g^{\prime \prime \prime}, g^{\prime} g^{\prime \prime \prime}$ become the followings.

$$
\left\{\begin{array}{l}
g^{\prime}=f^{\prime} \frac{d x}{d t}=f^{\prime} \frac{1}{q x^{q-1}} \\
g^{\prime 2}=\frac{1}{q^{2}} f^{\prime 2} \frac{1}{x^{2(q-1)}} \\
g^{\prime \prime}=\frac{1}{q^{2}}\left(f^{\prime \prime} x^{-(2 q-2)}-(q-1) f x^{-(2 q-1)}\right) \\
g^{\prime \prime 2}=f^{\prime \prime 2} \frac{1}{q^{4}} \frac{1}{x^{2(2 q-2)}}-2 f^{\prime \prime} \frac{1}{q^{2}} \frac{1}{x^{2 q-2}} f^{\prime} \frac{q-1}{q^{2}} \frac{1}{x^{2 q-1}}+f^{\prime 2} \frac{(q-1)^{2}}{q^{4}} \frac{1}{x^{2(2 q-1)}} \\
g^{\prime \prime \prime}=\frac{1}{q^{3}}\left(\left(f^{\prime \prime \prime}+(2 q-1)(q-1) f^{\prime}\right) x^{-3(q-1)}-(3 q-3) f^{\prime \prime} x^{-3(3 q-2)}\right) \\
g^{\prime} g^{\prime \prime \prime}=\frac{1}{q^{4}} f^{\prime}\left(\left(f^{\prime \prime \prime}+(2 q-1)(q-1) f^{\prime}\right) \frac{1}{x^{4 q-4}}-(3 q-3) \frac{f^{\prime \prime}}{x^{4 q-3}}\right)
\end{array}\right.
$$

Therefore we obtain

$$
x_{k+1}^{q}-\alpha^{q}=\frac{\left(\begin{array}{l}
\frac{1}{4} \frac{(q-1)^{2}}{q^{2}} f^{\prime 2} \frac{1}{x^{2 q}}-\frac{1}{q^{2}}(q-1) f f^{\prime \prime} \frac{1}{x^{2 q-1}} \\
+\frac{1}{q^{2}}\left(\frac{1}{4} f^{\prime \prime 2}-\frac{1}{6} f^{\prime}\left(f^{\prime \prime \prime}+(2 q-1)(q-1) f^{\prime}\right)\right) \frac{1}{x^{2 q-2}}
\end{array}\right)}{f^{\prime 2}}\left(x_{k}^{q}-\alpha^{q}\right)^{3}
$$


By lemma 3.2, we get

$$
x_{k+1}^{q}-\alpha^{q}=q \alpha^{q-1}\left(x_{k+1}-\alpha\right), x_{k}^{q}-\alpha^{q}=q \alpha^{q-1}\left(x_{k}-\alpha\right) .
$$

Therefore, formula (25) becomes

$$
\begin{aligned}
& q \alpha^{q-1}\left(x_{k+1}-\alpha\right) \\
& =\frac{\left(\begin{array}{l}
\frac{1}{4} \frac{(q-1)^{2}}{q^{2}} f^{\prime 2} \frac{1}{x^{2 q}}-\frac{1}{q^{2}}(q-1) f f^{\prime \prime} \frac{1}{x^{2 q-1}} \\
+\frac{1}{q^{2}}\left(\frac{1}{4} f^{\prime \prime 2}-\frac{1}{6} f^{\prime}\left(f^{\prime \prime \prime}+(2 q-1)(q-1) f^{\prime}\right)\right) \frac{1}{x^{2 q-2}}
\end{array}\right)}{f^{\prime 2}}\left(q \alpha^{q-1}\right)^{2} q \alpha^{q-1}\left(x_{k}-\alpha\right)^{3} .
\end{aligned}
$$

By changing the independent variable $x$ of the functions $f^{\prime}, f^{\prime \prime}, f^{\prime \prime \prime}$ and $x$ in numerator to $\alpha$, we obtain (21).

In case that $\alpha^{q}$ is $m$ multiple root, by (16), (1) and (20) we obtain

$$
\left\{\begin{array}{l}
t_{k+1}-\alpha^{q} \fallingdotseq\left(1-\frac{2}{m}\right)\left(t_{k}-\alpha^{q}\right) \\
x_{k+1}^{q}-\alpha^{q} \fallingdotseq\left(1-\frac{2}{m}\right)\left(x_{k}^{q}-\alpha^{q}\right) \\
x_{k+1}-\alpha \fallingdotseq\left(1-\frac{2}{m}\right)\left(x_{k}-\alpha\right)
\end{array}\right.
$$

Theorem 3.4. Let $\alpha(\neq 0)$ be a simple root of $f(x)=0$. Then a necessary and sufficient condition for the convergence to $\alpha$ of $q$-th power of $E H$-method is equal to or faster than Halley's method is that $q$ satisfies the following conditions.

$$
\left|1+\frac{(1-q) f^{\prime}(\alpha)\left[\left(\frac{1}{6}(2 q-1)+\frac{1-q}{4 \alpha^{2}}\right) f^{\prime}(\alpha)+\frac{f^{\prime \prime}(\alpha)}{\alpha}\right]}{\frac{1}{4} f^{\prime \prime}(\alpha)^{2}-\frac{1}{6} f^{\prime}(\alpha) f^{\prime \prime \prime}(\alpha)}\right| \leq 1
$$

That is

$$
0 \leq \frac{(q-1) f^{\prime}(\alpha)^{2}\left[\left(\frac{1}{3}-\frac{1}{4 \alpha^{2}}\right) q-\frac{1}{6}+\frac{1}{4 \alpha^{2}}+\frac{f^{\prime \prime}(\alpha)}{\alpha f^{\prime}(\alpha)}\right]}{\frac{1}{4} f^{\prime \prime}(\alpha)^{2}-\frac{1}{6} f^{\prime}(\alpha) f^{\prime \prime \prime}(\alpha)} \leq 2
$$

Proof. Compare the coefficient of $\left(x_{k}-\alpha\right)^{3}$ of the third-order convergence of $q$-th power of $E H$-method and that in the case of Halley's method. Then the necessary and sufficient condition is equivalent to the next formula.

$$
\frac{\left|\begin{array}{l}
\frac{1}{4} f^{\prime \prime}(\alpha)^{2}-\frac{1}{6} f^{\prime}(\alpha)\left(f^{\prime \prime \prime}(\alpha)+(2 q-1)(q-1) f^{\prime}(\alpha)\right) \\
+\frac{(q-1)^{2}}{4 \alpha^{2}} f^{\prime}(\alpha)^{2}-\frac{(q-1) f^{\prime}(\alpha) f^{\prime \prime}(\alpha)}{\alpha}
\end{array}\right|}{f^{\prime}(\alpha)^{2}} \leq \frac{\left|\frac{1}{4} f^{\prime \prime}(\alpha)^{2}-\frac{1}{6} f^{\prime}(\alpha) f^{\prime \prime \prime}(\alpha)\right|}{f^{\prime}(\alpha)^{2}}
$$


The formula (29) is obtained from this.

Corollary 3.5. (1) If $f^{\prime \prime}(\alpha)=0, f^{\prime \prime \prime}(\alpha) \neq 0$ then (30) becomes

$$
0 \leq \frac{(1-q) f^{\prime}(\alpha)\left[\left(2-\frac{3}{2 \alpha^{2}}\right) q-1+\frac{3}{2 \alpha^{2}}\right]}{f^{\prime \prime \prime}(\alpha)} \leq 2 .
$$

(2) If $f^{\prime \prime}(\alpha) \neq 0, f^{\prime \prime \prime}(\alpha)=0$ then (30) becomes

$$
0 \leq \frac{(q-1) f^{\prime}(\alpha)^{2}\left[\left(\frac{4}{3}-\frac{1}{\alpha^{2}}\right) q-\frac{2}{3}+\frac{1}{\alpha^{2}}+\frac{4 f^{\prime \prime}(\alpha)}{\alpha f^{\prime}(\alpha)}\right]}{f^{\prime \prime}(\alpha)^{2}} \leq 2 .
$$

We transform the equation $f(x)=0$ into $h(x)=0$. That is, two equations have the same root. $r$-th power of $E H$-method for $h(x)$ is

$$
x_{k+1}^{r}=x_{k}^{r}-q x_{k}^{r-1} \frac{h\left(x_{k}\right)}{h^{\prime}\left(x_{k}\right)-\frac{1}{2} \frac{h\left(x_{k}\right)\left(\frac{1}{q x_{k}^{r-1}} h^{\prime \prime}\left(x_{k}\right)+(1-r) \frac{1}{x_{k}} h^{\prime}\left(x_{k}\right)\right)}{h^{\prime}\left(x_{k}\right)}}
$$

and if $\alpha(\neq 0)$ is a simple root, then it becomes the third-order convergence (35).

$$
x_{k+1}-\alpha=\frac{\left[\begin{array}{l}
\frac{(r-1)^{2}}{4 \alpha^{2}} h^{\prime}(\alpha)^{2}-\frac{(r-1) h^{\prime}(\alpha) h^{\prime \prime}(\alpha)}{\alpha}+\frac{1}{4} h^{\prime \prime}(\alpha)^{2} \\
-\frac{1}{6} h^{\prime}(\alpha)\left(h^{\prime \prime \prime}(\alpha)+(2 r-1)(r-1) h^{\prime}(\alpha)\right)
\end{array}\right]}{h^{\prime}(\alpha)^{2}}\left(x_{k}-\alpha\right)^{3}
$$

We get the following by comparing the coefficient of $\left(x_{k}-\alpha\right)^{3}$ of formula (21) and (35).

Proposition 3.6. Let $f(\alpha)=h(\alpha)=0$, and $\alpha(\neq 0)$ a simple root. Then a necessary and sufficient condition for the convergence to $\alpha$ of $q$-th power of $E H$-method (Extended Halley's method) (13) of $f(x)$ to be equal to or faster than that $r$-th power of $E H$-method (34) of $h(x)$ is that the real numbers $q$ and $r$ satisfy the following condition (36).

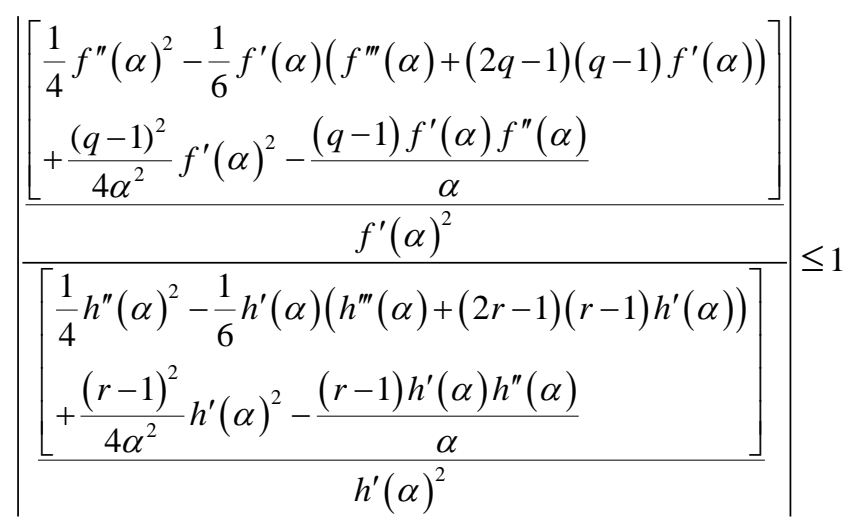


Theorem 3.7. Let $\alpha(\neq 0)$ be a simple root for $f(x)=0$, i.e., $f^{\prime}(\alpha) \neq 0$. Inequality (29) is represented by the second derivative

$$
g^{\prime \prime}(t)=g^{\prime \prime}\left(x^{q}\right)=\frac{x f^{\prime \prime}(x)+(1-q) f^{\prime}(x)}{q^{2} x^{2 q-1}}(>0(<0))
$$

which distinguishes the convex-concave of the curve $g(x)$. It shows the next complicated inequalities (38), (39).

(i) $\frac{(1-q)}{\alpha} f^{\prime}(\alpha)>0$

$$
\begin{aligned}
& \frac{\alpha}{(1-q) f^{\prime}(\alpha)} \frac{1}{\left(q \alpha^{q-1}\right)^{2}}\left[-\left(\frac{1}{4} f^{\prime \prime}(\alpha)^{2}-\frac{1}{6} f^{\prime}(\alpha) f^{\prime \prime \prime}(\alpha)\right)\right. \\
& \left.-\left|\frac{1}{4} f^{\prime \prime}(\alpha)^{2}-\frac{1}{6} f^{\prime}(\alpha) f^{\prime \prime \prime}(\alpha)\right|-\left(-\frac{3}{4} \frac{1-q}{\alpha^{2}}+\frac{2 q-1}{6}\right)(1-q) f^{\prime}(\alpha)^{2}\right] \\
& \leq \frac{f^{\prime \prime}(\alpha)+\frac{(1-q) f^{\prime}(\alpha)}{\alpha}}{\left(q \alpha^{q-1}\right)^{2}} \\
& \leq \frac{\alpha}{(1-q) f^{\prime}(\alpha)} \frac{1}{\left(q \alpha^{q-1}\right)^{2}}\left[-\left(\frac{1}{4} f^{\prime \prime}(\alpha)^{2}-\frac{1}{6} f^{\prime}(\alpha) f^{\prime \prime \prime}(\alpha)\right)\right. \\
& \left.\quad+\left|\frac{1}{4} f^{\prime \prime}(\alpha)^{2}-\frac{1}{6} f^{\prime}(\alpha) f^{\prime \prime \prime}(\alpha)\right|-\left(-\frac{3}{4} \frac{1-q}{\alpha^{2}}+\frac{2 q-1}{6}\right)(1-q) f^{\prime}(\alpha)^{2}\right]
\end{aligned}
$$

(ii) $\frac{(1-q)}{\alpha} f^{\prime}(\alpha)<0$

$$
\begin{aligned}
& \frac{\alpha}{(1-q) f^{\prime}(\alpha)} \frac{1}{\left(q \alpha^{q-1}\right)^{2}}\left[-\left(\frac{1}{4} f^{\prime \prime}(\alpha)^{2}-\frac{1}{6} f^{\prime}(\alpha) f^{\prime \prime \prime}(\alpha)\right)\right. \\
& +\left|\frac{1}{4} f^{\prime \prime}(\alpha)^{2}-\frac{1}{6} f^{\prime}(\alpha) f^{\prime \prime \prime}(\alpha)\right|-\left(-\frac{3}{4} \frac{1-q}{\alpha^{2}}+\frac{2 q-1}{6}\right)(1-q) f^{\prime}(\alpha)^{2} \\
& \leq \frac{f^{\prime \prime}(\alpha)+\frac{(1-q) f^{\prime}(\alpha)}{\alpha}}{\left(q \alpha^{q-1}\right)^{2}} \\
& \leq \frac{\alpha}{(1-q) f^{\prime}(\alpha)} \frac{1}{\left(q \alpha^{q-1}\right)^{2}}\left[-\left(\frac{1}{4} f^{\prime \prime}(\alpha)^{2}-\frac{1}{6} f^{\prime}(\alpha) f^{\prime \prime \prime}(\alpha)\right)\right. \\
& \left.-\left|\frac{1}{4} f^{\prime \prime}(\alpha)^{2}-\frac{1}{6} f^{\prime}(\alpha) f^{\prime \prime \prime}(\alpha)\right|-\left(-\frac{3}{4} \frac{1-q}{\alpha^{2}}+\frac{2 q-1}{6}\right)(1-q) f^{\prime}(\alpha)^{2}\right]
\end{aligned}
$$

Proof. We lead inequalities (38), (39) from (29). Let

$$
\begin{aligned}
& A=\frac{1}{4} f^{\prime \prime}(\alpha)^{2}-\frac{1}{6} f^{\prime}(\alpha) f^{\prime \prime \prime}(\alpha), \\
& B=\frac{(1-q)^{2}}{4 \alpha^{2}} f^{\prime}(\alpha)^{2}+\frac{(1-q) f^{\prime}(\alpha) f^{\prime \prime}(\alpha)}{\alpha}+\frac{1}{6}(2 q-1)(1-q) f^{\prime}(\alpha)^{2} .
\end{aligned}
$$

Then inequality (29) becomes 


$$
\begin{gathered}
\left|1+\frac{B}{A}\right| \leq 1 \\
-A-|A| \leq B \leq-A+|| A \mid .
\end{gathered}
$$

We transform the formula $B$.

$$
\begin{aligned}
B= & \left(\frac{1-q}{4 \alpha^{2}} f^{\prime}(\alpha)+\frac{f^{\prime \prime}(\alpha)}{\alpha}+\frac{1}{6}(2 q-1) f^{\prime}(\alpha)\right)(1-q) f^{\prime}(\alpha) \\
= & \left(\frac{(1-q) f^{\prime}(\alpha)+\alpha f^{\prime \prime}(\alpha)}{q^{2} \alpha^{2 q-1}}\right) \frac{1}{\alpha}(1-q) f^{\prime}(\alpha) q^{2} \alpha^{2 q-2} \\
& +\left(-\frac{3}{4} \frac{1-q}{\alpha^{2}} f^{\prime}(\alpha)+\frac{1}{6}(2 q-1) f^{\prime}(\alpha)\right)(1-q) f^{\prime}(\alpha)
\end{aligned}
$$

Therefore inequality (29) becomes (44).

$$
\begin{aligned}
& -A-|A| \\
\leq & \left(\frac{(1-q) f^{\prime}(\alpha)+\alpha f^{\prime \prime}(\alpha)}{q^{2} \alpha^{2 q-1}}\right) \frac{1}{\alpha}(1-q) f^{\prime}(\alpha) q^{2} \alpha^{2 q-2} \\
& +\left(-\frac{3}{4} \frac{1-q}{\alpha^{2}} f^{\prime}(\alpha)+\frac{1}{6}(2 q-1) f^{\prime}(\alpha)\right)(1-q) f^{\prime}(\alpha) \\
\leq & -A+|A|
\end{aligned}
$$

Furthermore, we transform the inequality.

$$
\begin{aligned}
& \frac{1}{q^{2} \alpha^{2 q-2}}\left[-A-|A|-\left(-\frac{3}{4} \frac{1-q}{\alpha^{2}} f^{\prime}(\alpha)+\frac{1}{6}(2 q-1) f^{\prime}(\alpha)\right)(1-q) f^{\prime}(\alpha)\right] \\
& \leq\left(\frac{(1-q) f^{\prime}(\alpha)+\alpha f^{\prime \prime}(\alpha)}{q^{2} \alpha^{2 q-1}}\right) \frac{1}{\alpha}(1-q) f^{\prime}(\alpha) \\
& \leq \frac{1}{q^{2} \alpha^{2 q-2}}\left[-A+|A|-\left(-\frac{3}{4} \frac{1-q}{\alpha^{2}} f^{\prime}(\alpha)+\frac{1}{6}(2 q-1) f^{\prime}(\alpha)\right)(1-q) f^{\prime}(\alpha)\right]
\end{aligned}
$$

From (45), we get (38), (39) according to plus, minus number of $(1-q) f^{\prime}(\alpha) / \alpha$ respectively.

Theorem 3.8. Let the condition be the same as the above Theorem. Inequality (29) is represented by the curvature

$$
\mu_{q}(t)=\frac{g^{\prime \prime}(t)}{\left(1+g^{\prime}(t)^{2}\right)^{3 / 2}}=\mu_{q}\left(x^{q}\right)=\frac{f^{\prime \prime}(x)+\frac{(1-q) f^{\prime}(x)}{x}}{\left(q x^{q-1}\right)^{2}\left(1+\left(\frac{f^{\prime}(x)}{q x^{q-1}}\right)^{2}\right)^{3 / 2}} .
$$

Those are the next complicated inequalities (47) and (48).

(i) $\frac{(1-q)}{\alpha} f^{\prime}(\alpha)>0$ 


$$
\begin{aligned}
& \frac{\alpha}{(1-q) f^{\prime}(\alpha)} \frac{1}{\left(q \alpha^{q-1}\right)^{2}\left(1+\left(\frac{f^{\prime}(\alpha)}{q \alpha^{q-1}}\right)^{2}\right)^{\frac{3}{2}}}\left[-\left(\frac{1}{4} f^{\prime \prime}(\alpha)^{2}-\frac{1}{6} f^{\prime}(\alpha) f^{\prime \prime \prime}(\alpha)\right)\right. \\
& \left.-\left|\frac{1}{4} f^{\prime \prime}(\alpha)^{2}-\frac{1}{6} f^{\prime}(\alpha) f^{\prime \prime \prime}(\alpha)\right|-\left(-\frac{3}{4} \frac{1-q}{\alpha^{2}}+\frac{1}{6}(2 q-1)\right)(1-q) f^{\prime}(\alpha)^{2}\right] \\
& \leq \frac{f^{\prime \prime}(\alpha)+\frac{(1-q) f^{\prime}(\alpha)}{\alpha}}{\leq} \frac{\left(q \alpha^{q-1}\right)^{2}\left(1+\left(\frac{f^{\prime}(\alpha)}{q \alpha^{q-1}}\right)^{2}\right)^{\frac{3}{2}}}{(1-q) f^{\prime}(\alpha)} \frac{1}{\left(q \alpha^{q-1}\right)^{2}\left(1+\left(\frac{f^{\prime}(\alpha)}{q \alpha^{q-1}}\right)^{2}\right)^{\frac{3}{2}}}\left[-\left(\frac{1}{4} f^{\prime \prime}(\alpha)^{2}-\frac{1}{6} f^{\prime}(\alpha) f^{\prime \prime \prime}(\alpha)\right)\right. \\
& \left.+\left|\frac{1}{4} f^{\prime \prime}(\alpha)^{2}-\frac{1}{6} f^{\prime}(\alpha) f^{\prime \prime \prime}(\alpha)\right|-\left(-\frac{3}{4} \frac{1-q}{\alpha^{2}}+\frac{1}{6}(2 q-1)\right)(1-q) f^{\prime}(\alpha)^{2}\right]
\end{aligned}
$$

(ii) $\frac{(1-q)}{\alpha} f^{\prime}(\alpha)<0$

$$
\begin{aligned}
& \frac{\alpha}{(1-q) f^{\prime}(\alpha)} \frac{1}{\left(q \alpha^{q-1}\right)^{2}\left(1+\left(\frac{f^{\prime}(\alpha)}{q \alpha^{q-1}}\right)^{2}\right)^{\frac{3}{2}}}\left[-\left(\frac{1}{4} f^{\prime \prime}(\alpha)^{2}-\frac{1}{6} f^{\prime}(\alpha) f^{\prime \prime \prime}(\alpha)\right)\right. \\
& \left.+\left|\frac{1}{4} f^{\prime \prime}(\alpha)^{2}-\frac{1}{6} f^{\prime}(\alpha) f^{\prime \prime \prime}(\alpha)\right|-\left(-\frac{3}{4} \frac{1-q}{\alpha^{2}}+\frac{1}{6}(2 q-1)\right)(1-q) f^{\prime}(\alpha)^{2}\right] \\
& \leq \frac{f^{\prime \prime}(\alpha)+\frac{(1-q) f^{\prime}(\alpha)}{\alpha}}{\left(q \alpha^{q-1}\right)^{2}\left(1+\left(\frac{f^{\prime}(\alpha)}{q \alpha^{q-1}}\right)^{2}\right)^{\frac{3}{2}}} \\
& \leq \frac{\alpha}{(1-q) f^{\prime}(\alpha)} \frac{1}{\left(q \alpha^{q-1}\right)^{2}\left(1+\left(\frac{f^{\prime}(\alpha)}{q \alpha^{q-1}}\right)^{2}\right)^{\frac{3}{2}}}\left[-\left(\frac{1}{4} f^{\prime \prime}(\alpha)^{2}-\frac{1}{6} f^{\prime}(\alpha) f^{\prime \prime \prime}(\alpha)\right)\right. \\
& \left.-\left|\frac{1}{4} f^{\prime \prime}(\alpha)^{2}-\frac{1}{6} f^{\prime}(\alpha) f^{\prime \prime \prime}(\alpha)\right|-\left(-\frac{3}{4} \frac{1-q}{\alpha^{2}}+\frac{1}{6}(2 q-1)\right)(1-q) f^{\prime}(\alpha)^{2}\right]
\end{aligned}
$$

Proof. We get (47), (48) by dividing formula (38), (39) in $\left(1+\left(f^{\prime}(x) / q x^{q-1}\right)^{2}\right)^{3 / 2}$, respectively.

\section{Convergence Comparisons by the Numerical Calculations of Halley's Method and Extensions of Halley's Method}

We perform numerical calculations by the calculation formula (14) in the standard 
format in Excel 2013 of Microsoft. We perform numerical calculations for various equations such as $n$-th order equations ( $n \geq 2)$, equations of trigonometric, exponential, logarithmic function, respectively.

In the examples of the followings, there are cases where some numerical calculations do not fit in with the inequality (30) a little. Those are probably due to the formula (21) the approximate formula, choosing the initial value $x_{0}$, and the accuracy of using the standard format in Excel is insufficient. However, the results to fit the theories generally have been obtained.

Example 4.1. A quadratic equation

$$
f(x)=(x-1)(x-2)=x^{2}-3 x+2=0
$$

The roots of (49) are $\alpha=1,2$. Because $f^{\prime}(x)=2 x-3, f^{\prime \prime}(x)=2, f^{\prime \prime \prime}(x)=0$, in case of $\alpha=1$, condition (33) becomes

$$
0 \leq(q-1)\left(\frac{1}{12} q+\frac{1}{12}-2\right) \leq 2 \Leftrightarrow \text { about }-0.041 \leq q \leq 1,23 \leq q \leq 24.042 .
$$

We choose real numbers $q$ and initial values $x_{0}$ such as Table 1, Table 2, and do numerical computations. We explain how to read Table 1. The first column represents the initial value $x_{0}$ and the absolute error, and the first row represents the real number $q$ of $x_{k}^{q}$.

Two numbers 1 and $1.11022 \mathrm{E}-16$ of intersection of two row and two column mean the following.

Number 1 indicates the number of iterations that Halley's method $(q=1)$ to converge to the root $1.1 .11022 \mathrm{E}-16$ indicates the absolute error |the value $x_{1}=1$ of the convergence of the numerical calculation-root 1|. If two iteration numbers are the same for the same initial value $x_{0}$, then we evaluate the convergences by the absolute errors. In the Table 1, Table 2, all $q$-th power of $E H$-method (Extension of Halley's method) converge in root 1 at iteration number $k=1$. But, for the same initial value $x_{0}$, each column of $E H$-method $(q \neq 1)$ has the absolute errors (at least one) that are equal to or smaller than Halley's method $(q=1)$ in the ranges of (50).

We confirm Theorem 3.7. Because $(1-q) f^{\prime}(1) / \alpha=q-1<0$ in $-0.041 \leq q \leq 1$, inequality (39) is applied. In this case (39) becomes the following inequality.

$$
\frac{3}{4}(q-1)+\frac{1}{6}(2 q-1) \leq q+1 \leq \frac{1}{q-1}\left[-2+\frac{3}{4}(q-1)^{2}+\frac{1}{6}(2 q-1)(q-1)\right]
$$

The results are Table 3. The range of $q$ which satisfies (51) becomes $-0.041 \leq q \leq 1$.

Example 4.2. A cubic equation

$$
f(x)=x^{3}-8=0
$$

Because the root of (52) is 2 , the condition (30) becomes

$$
0 \leq(q-1)[0.125(13 q-5)+3)] \leq 2 \Leftrightarrow-1.8875 \leq q \leq-1.462,1 \leq q \leq 1.4262 .
$$

We choose real numbers $q$ and initial values $x_{0}$ such as Table 4, Table 5, and do numerical computations. All iteration numbers are 2 or 3 . But, for the same initial value 
Table 1. Calculations of (14) for root $1,-0.041 \leq q \leq 1$.

\begin{tabular}{|c|c|c|c|c|c|c|c|c|c|c|}
\hline $\begin{array}{l}x_{0} \\
\longrightarrow\end{array}$ & 1 & -0.041 & -0.025 & -0.01 & 0.1 & 0.2 & 0.4 & 0.6 & 0.8 & 0.9 \\
\hline 0.999999993 & $k=1$ & 1 & 1 & 1 & 1 & 1 & 1 & 1 & 1 & 1 \\
\hline Absolute errors & $1.11022 \mathrm{E}-16$ & 0 & 0 & 0 & 0 & 0 & 0 & $2.22045 \mathrm{E}-16$ & $1.11022 \mathrm{E}-16$ & $2.22045 \mathrm{E}-16$ \\
\hline 1.000000003 & 1 & 1 & 1 & 1 & 1 & 1 & 1 & 1 & 1 & 1 \\
\hline Absolute errors & $2.22045 \mathrm{E}-16$ & 0 & 0 & 0 & 0 & 0 & $4.44089 \mathrm{E}-16$ & 0 & $2.22045 \mathrm{E}-16$ & $2.22045 \mathrm{E}-16$ \\
\hline
\end{tabular}

Table 2. Calculations of (14) for root $1,23 \leq q \leq 24.042$.

\begin{tabular}{|c|c|c|c|c|c|c|c|c|}
\hline $\begin{array}{l}x_{0} \\
\longrightarrow\end{array}$ & 1 & 23.1 & 23.3 & 23.5 & 23.7 & 23.9 & 24.042 & 24.1 \\
\hline 0.999999992 & 1 & 1 & 1 & 1 & 1 & 1 & 1 & 1 \\
\hline Absolute errors & $2.22045 \mathrm{E}-16$ & 0 & 0 & 0 & 0 & 0 & 0 & 0 \\
\hline 1.000000015 & 1 & 1 & 1 & 1 & 1 & 1 & 1 & 1 \\
\hline Absolute errors & $2.22045 \mathrm{E}-16$ & 0 & 0 & 0 & 0 & 0 & 0 & 0 \\
\hline
\end{tabular}

Table 3. Calculations of (51) for root $1,-0.041 \leq q \leq 1$.

\begin{tabular}{|c|c|c|c|c|}
\hline$q$ & $(1-q) f(\alpha) / \alpha=q-1$ & Left-hand side of $g^{\prime \prime}(t)$ & $g^{\prime \prime}(t)=q+1$ & Right-hand side of $g^{\prime \prime}(t)$ \\
\hline-0.4 & -1.4 & -1.35 & 0.6 & 0.078571429 \\
\hline-0.3 & -1.3 & -1.241666667 & 0.7 & 0.296794872 \\
\hline-0.2 & -1.2 & -1.133333333 & 0.8 & 0.533333333 \\
\hline-0.1 & -1.1 & -1.025 & 0.9 & 0.793181818 \\
\hline-0.042 & -1.042 & -0.962166667 & 0.958 & 0.95721913 \\
\hline-0.041 & -1.041 & -0.961083333 & 0.959 & 0.960146254 \\
\hline 0 & -1 & -0.916666667 & 1 & 1.083333333 \\
\hline 0.1 & -0.9 & -0.808333333 & 1.1 & 1.413888889 \\
\hline 0.2 & -0.8 & -0.7 & 1.2 & 1.8 \\
\hline 0.3 & -0.7 & -0.591666667 & 1.3 & 2.26547619 \\
\hline 0.4 & -0.6 & -0.483333333 & 1.4 & 2.85 \\
\hline 0.5 & -0.5 & -0.375 & 1.5 & 3.625 \\
\hline 0.6 & -0.4 & -0.266666667 & 1.6 & 4.733333333 \\
\hline 0.7 & -0.3 & -0.158333333 & 1.7 & 6.508333333 \\
\hline 0.8 & -0.2 & -0.05 & 1.8 & 9.95 \\
\hline 0.9 & -0.1 & 0.058333333 & 1.9 & 20.05833333 \\
\hline 0.9999 & $-1 \mathrm{E}-04$ & 0.166558333 & 1.9999 & 20000.16656 \\
\hline 1 & 0 & \#DIV/0! & 2 & \#DIV/0! \\
\hline 1.001 & 0.001 & 0.16775 & 2.001 & -1999.83225 \\
\hline 1.1 & 0.1 & 0.275 & 2.1 & -19.725 \\
\hline 1.2 & 0.2 & 0.383333333 & 2.2 & -9.616666667 \\
\hline
\end{tabular}


Table 4. Calculations of (14) for root $2,-1.8875 \leq q \leq-1.462$.

\begin{tabular}{|c|c|c|c|c|c|c|}
\hline$q$ & -1.8875 & -1.7 & -1.6 & -1.5 & -1.462 & 1 \\
\hline 1.999 & 2 & 2 & 2 & 2 & 2 & 2 \\
\hline Absolute errors & $5.28466 \mathrm{E}-14$ & $5.28466 \mathrm{E}-14$ & $5.24025 \mathrm{E}-14$ & $5.28466 \mathrm{E}-14$ & $5.28466 \mathrm{E}-14$ & $5.28466 \mathrm{E}-14$ \\
\hline 2.05 & 3 & 3 & 3 & 3 & 3 & 3 \\
\hline Absolute errors & $4.26326 \mathrm{E}-14$ & $4.26326 \mathrm{E}-14$ & $4.35207 \mathrm{E}-14$ & $4.30767 \mathrm{E}-14$ & $4.35207 \mathrm{E}-14$ & $4.39648 \mathrm{E}-14$ \\
\hline 2.1 & 3 & 3 & 3 & 3 & 3 & 3 \\
\hline Absolute errors & $1.12594 \mathrm{E}-11$ & $1.14033 \mathrm{E}-11$ & $1.14744 \mathrm{E}-11$ & $1.15419 \mathrm{E}-11$ & $1.15663 \mathrm{E}-11$ & $1.18154 \mathrm{E}-11$ \\
\hline 2.15 & 3 & 3 & 3 & 3 & 3 & 3 \\
\hline Absolute errors & $2.92275 \mathrm{E}-10$ & $2.97491 \mathrm{E}-10$ & $3.00076 \mathrm{E}-10$ & $3.02517 \mathrm{E}-10$ & $3.03407 \mathrm{E}-10$ & $3.12511 \mathrm{E}-10$ \\
\hline
\end{tabular}

Table 5. Calculations of (14) for root $2,1 \leq q \leq 1.4262$.

\begin{tabular}{|c|c|c|c|c|c|c|}
\hline $\begin{array}{l} \\
x_{0}\end{array}$ & 1 & 1.001 & 1.1 & 1.2 & 1.3 & 1.4262 \\
\hline 1.999 & 2 & 2 & 2 & 2 & 2 & 2 \\
\hline Absolute errors & $5.28466 \mathrm{E}-14$ & $5.24025 \mathrm{E}-14$ & $5.24025 \mathrm{E}-14$ & $5.28466 \mathrm{E}-14$ & $5.24025 \mathrm{E}-14$ & $5.24025 \mathrm{E}-14$ \\
\hline 2.05 & 3 & 3 & 3 & 3 & 3 & 3 \\
\hline Absolute errors & $4.39648 \mathrm{E}-14$ & $4.39648 \mathrm{E}-14$ & $4.35207 \mathrm{E}-14$ & $4.35207 \mathrm{E}-14$ & $4.35207 \mathrm{E}-14$ & $4.30767 \mathrm{E}-14$ \\
\hline 2.1 & 3 & 3 & 3 & 3 & 3 & 3 \\
\hline Absolute errors & $1.18154 \mathrm{E}-11$ & $1.18154 \mathrm{E}-11$ & $1.17693 \mathrm{E}-11$ & $1.17186 \mathrm{E}-11$ & $1.1664 \mathrm{E}-11$ & $1.15885 \mathrm{E}-11$ \\
\hline 2.15 & 3 & 3 & 3 & 3 & 3 & 3 \\
\hline Absolute errors & $3.12511 \mathrm{E}-10$ & $3.12495 \mathrm{E}-10$ & $3.10816 \mathrm{E}-10$ & $3.08966 \mathrm{E}-10$ & $3.06965 \mathrm{E}-10$ & $3.04226 \mathrm{E}-10$ \\
\hline
\end{tabular}

$X_{0}$, each column of $E H$-method $(q \neq 1)$ has the absolute errors (at least one) that are equal to or smaller than Halley's method $(q=1)$ in the ranges of (53).

Example 4.3. A cubic equation

$$
f(x)=(x-1)(x-2)(x-3)=0
$$

In case of the root 1 , the condition (30) becomes

$$
0 \leq \frac{1}{21}(q-1)(q-35) \leq 2 \Leftrightarrow-0.1934 \leq q \leq 1,35 \leq q \leq 36.1934 .
$$

We choose real numbers $q$ and initial values $x_{0}$ such as Table 6, Table 7, and do numerical computations. Each initial value $x_{0}$, iteration number of $E H$-method $(q \neq 1)$ and Halley's method $(q=1)$ are the same. But, for the same initial value $x_{0}$, each column of $E H$-method $(q \neq 1)$ has the absolute errors (at least one) that are smaller than Halley's method $(q=1)$ in the ranges of (55).

Example 4.4.

$$
f(x)=\sin x=0
$$

The roots of (56) are $n \pi(n=0, \pm 1, \pm 2, \cdots)$. The condition (30) becomes 


$$
0 \leq(q-1)\left(2 q-1-\frac{3(q-1)}{2(n \pi)^{2}}\right) \leq 2(n= \pm 1, \pm 2, \cdots)
$$

If we take the root in $\pi \fallingdotseq 3.141592654(n=1)$, then (57) becomes

$$
0 \leq(q-1)\left(2 q-1-\frac{3}{2} \frac{q-1}{\pi^{2}}\right) \leq 2 \Leftrightarrow-0.3455 \leq q \leq 0.458,1 \leq q \leq 1.8043 .
$$

We do numerical computations for the real numbers $q$ and initial values $x_{0}$ in Table 8, Table 9. All iteration numbers in Table 8 are 1. But, for the same initial value $x_{0}$, each column of EH-method $(q \neq 1)$ has the absolute errors (at least one) that are smaller than Halley's method $(q=1)$ in $-0.3455 \leq q \leq 0.458$. In case of $1 \leq q \leq 1.8043$, number of iterations of $E H$-methods $(q \neq 1)$ are small than Halley's method.

\begin{tabular}{|c|c|c|c|c|c|c|c|}
\hline$q$ & -0.1934 & 0.1 & 0.3 & 0.5 & 0.7 & 0.99 & 1 \\
\hline 0.875 & 3 & 3 & 3 & 3 & 3 & 3 & 3 \\
\hline Absolute error & 0 & 0 & 0 & $4.44089 \mathrm{E}-16$ & $2.22045 \mathrm{E}-16$ & $2.22045 \mathrm{E}-16$ & $4.44089 \mathrm{E}-16$ \\
\hline 0.99999741 & 1 & 1 & 1 & 1 & 1 & 1 & 1 \\
\hline Absolute error & 0 & 0 & 0 & $2.22045 \mathrm{E}-16$ & $1.11022 \mathrm{E}-16$ & $2.22045 \mathrm{E}-16$ & $2.22045 \mathrm{E}-16$ \\
\hline 1.000001 & 1 & 1 & 1 & 1 & 1 & 1 & 1 \\
\hline Absolute error & $4.44089 \mathrm{E}-16$ & 0 & 0 & $4.44089 \mathrm{E}-16$ & $2.22045 \mathrm{E}-16$ & $2.22045 \mathrm{E}-16$ & $4.44089 \mathrm{E}-16$ \\
\hline 1.1 & 3 & 3 & 3 & 3 & 3 & 3 & 3 \\
\hline Absolute error & $1.11022 \mathrm{E}-15$ & 0 & $3.33067 \mathrm{E}-16$ & 0 & $1.11022 \mathrm{E}-16$ & $2.22045 \mathrm{E}-16$ & $3.33067 \mathrm{E}-16$ \\
\hline
\end{tabular}

Table 6. Calculations of (14) for root $1,-0.1934 \leq q \leq 1$.

\begin{tabular}{|c|c|c|c|c|c|c|c|c|}
\hline $\begin{array}{ll}x_{0} & q\end{array}$ & 1 & 34 & 35 & 35.2 & 35.4 & 35.6 & 35.8 & 36.1934 \\
\hline 0.999999256 & 1 & 1 & 1 & 1 & 1 & 1 & 1 & 1 \\
\hline Absolute error & $5.55112 \mathrm{E}-16$ & $4.44089 \mathrm{E}-16$ & $4.44089 \mathrm{E}-16$ & $4.44089 \mathrm{E}-16$ & $4.44089 \mathrm{E}-16$ & $4.44089 \mathrm{E}-16$ & $4.44089 \mathrm{E}-16$ & $4.44089 \mathrm{E}-16$ \\
\hline 1.000001 & 1 & 1 & 1 & 1 & 1 & 1 & 1 & 1 \\
\hline Absolute error & $4.44089 \mathrm{E}-16$ & $2.22045 \mathrm{E}-16$ & $2.22045 \mathrm{E}-16$ & $2.22045 \mathrm{E}-16$ & $2.22045 \mathrm{E}-16$ & $2.22045 \mathrm{E}-16$ & $2.22045 \mathrm{E}-16$ & $2.22045 \mathrm{E}-16$ \\
\hline
\end{tabular}

Table 7. Calculations of (14) for root $1,35 \leq q \leq 36$.

\begin{tabular}{|c|c|c|c|c|c|c|c|}
\hline $\begin{array}{l} \\
x_{0}\end{array}$ & -0.3455 & -0.3 & -0.1 & 0.1 & 0.3 & 0.458 & 1 \\
\hline 3.1415 & 1 & 1 & 1 & 1 & 1 & 1 & 1 \\
\hline Absolute errors & $4.04578 \mathrm{E}-10$ & $4.04076 \mathrm{E}-10$ & $3.95939 \mathrm{E}-10$ & $4.21089 \mathrm{E}-10$ & $4.12902 \mathrm{E}-10$ & $4.11561 \mathrm{E}-10$ & $4.10339 \mathrm{E}-10$ \\
\hline 3.141585 & 1 & 1 & 1 & 1 & 1 & 1 & 1 \\
\hline Absolute errors & $4.10203 \mathrm{E}-10$ & $4.10203 \mathrm{E}-10$ & $4.10198 \mathrm{E}-10$ & $4.10209 \mathrm{E}-10$ & $4.10208 \mathrm{E}-10$ & $4.10208 \mathrm{E}-10$ & $4.10207 \mathrm{E}-10$ \\
\hline 3.1417 & 1 & 1 & 1 & 1 & 1 & 1 & 1 \\
\hline Absolute errors & $4.18962 \mathrm{E}-10$ & $4.19741 \mathrm{E}-10$ & $4.32396 \mathrm{E}-10$ & $4.06015 \mathrm{E}-10$ & $3.93276 \mathrm{E}-10$ & $4.08099 \mathrm{E}-10$ & $4.1 \mathrm{E}-10$ \\
\hline
\end{tabular}

Table 8. Calculations of (14) for root $\pi,-0.3455 \leq q \leq 0.458$. 


\section{Example 4.5.}

$$
f(x)=\tan x=0
$$

The roots of (59) are $n \pi(n=0, \pm 1, \pm 2, \cdots)$. The condition (30) becomes

$$
0 \leq-\frac{1}{2}(q-1)\left[\left(2-\frac{3}{2(n \pi)^{2}}\right) q-1+\frac{3}{2(n \pi)^{2}}\right] \leq 2 \quad(n= \pm 1, \pm 2, \cdots) .
$$

If we take the root in $\pi \fallingdotseq 3.141592654(n=1)$, then (60) becomes

$$
0 \leq-\frac{1}{2}(q-1)\left[\left(2-\frac{3}{2 \pi^{2}}\right) q-1+\frac{3}{2 \pi^{2}}\right] \leq 2 \Leftrightarrow 0.46 \leq q \leq 1 .
$$

Table 10 gives numerical computations. In case of $x_{0}=3.142, E H$-methods $(q \neq 1)$ have better approximate degrees than Halley's method $(q=1)$ in $0.46 \leq q \leq 1$.

Example 4.6.

$$
f(x)=e^{x}-e=0 \quad(e: \text { Napier's constant })
$$

The root of (62) is 1 . The condition (30) becomes

$$
0 \leq(q-1)(q+13) \leq 2 \Leftrightarrow-13.14142 \leq q \leq-13,1 \leq q \leq 1.14142 .
$$

Table 11 and Table 12 give the numerical values to almost adapt to Theorem 3.4.

\section{Example 4.7.}

$$
f(x)=\log x=0
$$

\begin{tabular}{|c|c|c|c|c|c|c|c|}
\hline$x_{0}$ & 0.8 & 1 & 1.2 & 1.4 & 1.6 & 1.8043 & 2 \\
\hline 3.14005 & 2 & 2 & 1 & 1 & 1 & 1 & 2 \\
\hline
\end{tabular}

The root of (64) is 1 . The condition (30) becomes

$$
0 \leq(1-q)(q-11) \leq 2 \Leftrightarrow 1 \leq q \leq 1.2041684,10.795834 \leq q \leq 11 .
$$

Table 13 and Table 14 give the numerical values to almost adapt to Theorem 3.4.

Table 9. Calculations of (14) for root $\pi, 1 \leq q \leq 1.8043$.

Table 10. Calculations of (14) for $\operatorname{root} \pi, 0.46 \leq q \leq 1$.

\begin{tabular}{cccccccc}
\hline & $q$ & 0.4 & 0.46 & 0.6 & 0.7 & 0.8 & 0.9 \\
$x_{0}$ & 2 & 2 & 2 & 2 & 2 & 2 \\
Absolute errors & $4.10207 \mathrm{E}-10$ & $4.10207 \mathrm{E}-10$ & $4.10207 \mathrm{E}-10$ & $4.10207 \mathrm{E}-10$ & $4.10206 \mathrm{E}-10$ & $4.10206 \mathrm{E}-10$ & $4.10207 \mathrm{E}-10$ \\
\hline 3.142 & 1 & 1 & 1 & 1 & 1 & 1 \\
Absolute errors & $2.8809 \mathrm{E}-11$ & $9.20344 \mathrm{E}-11$ & $1.86697 \mathrm{E}-10$ & $2.28724 \mathrm{E}-10$ & $2.58712 \mathrm{E}-10$ & $2.80826 \mathrm{E}-10$ & $2.97554 \mathrm{E}-10$ \\
\hline
\end{tabular}


Table 11. Calculations of (14) for $\operatorname{root} 1,-13.14142 \leq q \leq-13$.

\begin{tabular}{|c|c|c|c|c|c|c|c|c|}
\hline $\begin{array}{l} \\
x_{0}\end{array}$ & 1 & -13.2 & -13.14142 & -13.1 & -13.08 & -13.05 & -13.02 & -13 \\
\hline 0.999999998 & 1 & 1 & 1 & 1 & 1 & 1 & 1 & 1 \\
\hline 1.000000009 & 1 & 1 & 1 & 1 & 1 & 1 & 1 & 1 \\
\hline Absolute errors & $1.11022 \mathrm{E}-16$ & 0 & 0 & 0 & 0 & 0 & 0 & $2.22045 \mathrm{E}-16$ \\
\hline
\end{tabular}

Table 12. Calculations of (14) for $\operatorname{root} 1,1 \leq q \leq 1.14142$.

\begin{tabular}{|c|c|c|c|c|c|c|c|c|}
\hline $\begin{array}{l} \\
x_{0}\end{array}$ & 0.9 & 1 & 1.02 & 1.04 & 1.06 & 1.08 & 1.14142 & 1.15 \\
\hline 0.999999966 & 1 & 1 & 1 & 1 & 1 & 1 & 1 & 1 \\
\hline 1.00000004 & 1 & 1 & 1 & 1 & 1 & 1 & 1 & 1 \\
\hline Absolute errors & $2.22045 \mathrm{E}-16$ & $1.11022 \mathrm{E}-16$ & 0 & 0 & $1.11022 \mathrm{E}-16$ & $1.11022 \mathrm{E}-16$ & 0 & $1.11022 \mathrm{E}-16$ \\
\hline
\end{tabular}

Table 13. Calculations of (14) for root $1,1 \leq q \leq 1.2041684$.

\begin{tabular}{|c|c|c|c|c|c|c|c|c|}
\hline $\begin{array}{ll} & q \\
x_{0} & \end{array}$ & 0.9 & 1 & 1.04 & 1.08 & 1.12 & 1.16 & 1.2 & 1.2041684 \\
\hline 0.96 & 3 & 3 & 3 & 3 & 3 & 3 & 3 & 3 \\
\hline 1.01 & 3 & 3 & 3 & 3 & 3 & 3 & 3 & 3 \\
\hline Absolute errors & $2.22045 \mathrm{E}-16$ & $1.11022 \mathrm{E}-16$ & $1.11022 \mathrm{E}-16$ & $1.11022 \mathrm{E}^{-16}$ & 0 & $1.11022 \mathrm{E}^{-16}$ & 0 & $1.11022 \mathrm{E}-16$ \\
\hline
\end{tabular}

Table 14. Calculations of (14) for root $1,10.795834 \leq q \leq 11$.

\begin{tabular}{ccccccccc}
\hline & $q$ & 1 & 10 & 10.795834 & 10.9 & 10.94 & 10.98 & 11 \\
\hline \multicolumn{2}{c}{0.9} & 4 & 3 & 3 & 3 & 3 & 3 & 4 \\
\hline 1.15 & 4 & 4 & 4 & 4 & 4 & 4 & 4 \\
Absolute errors & $1.10389 \mathrm{E}-12$ & $1.66533 \mathrm{E}-15$ & $2.20934 \mathrm{E}-14$ & $8.53762 \mathrm{E}-14$ & $1.36779 \mathrm{E}-13$ & $2.14606 \mathrm{E}-13$ & $2.67009 \mathrm{E}-13$ \\
\hline
\end{tabular}

\section{Acknowledgements}

Dr. Hideko Nagasaka (Nihon University former professor) taught me numerical computations. I am deeply grateful to her.

\section{References}

[1] Murase, Y. (1673) Sanpoufutsudankai. In: Nishida, T., Ed., Kenseisha Co., Ltd., Tokyo. (In Japanese)

[2] Horiguchi, S. (2014) On Relations between the General Recurrence Formula of the Extension of Murase-Newton's Method (the Extension of Tsuchikura-Horiguchi's Method) and Horner's Method. Applied Mathematics, 5, 777-783. https://doi.org/10.4236/am.2014.54074

[3] Horiguchi, S (2016) The Formulas to Compare the Convergences of Newton's Method and 
the Extended Newton's Method (Tsuchikura-Horiguchi Method) and the Numerical Calculations. Applied Mathematics, 7, 40-60. https://doi.org/10.4236/am.2016.71004

[4] http://en.wikipedia.org/wiki/Halley's_method

[5] Nagasaka, H. (1980) Computer and Numerical Analysis (Japanese Title Keisanki to suuchikaiseki). Asakura Publishing Co., Ltd., Tokyo. (In Japanese)

Submit or recommend next manuscript to SCIRP and we will provide best service for you:

Accepting pre-submission inquiries through Email, Facebook, LinkedIn, Twitter, etc. A wide selection of journals (inclusive of 9 subjects, more than 200 journals)

Providing 24-hour high-quality service

User-friendly online submission system

Fair and swift peer-review system

Efficient typesetting and proofreading procedure

Display of the result of downloads and visits, as well as the number of cited articles Maximum dissemination of your research work

Submit your manuscript at: http://papersubmission.scirp.org/

Or contact am@scirp.org 